\title{
Pilot study on cardiogenic differentiation capability of rabbit mesenchymal stem cells
}

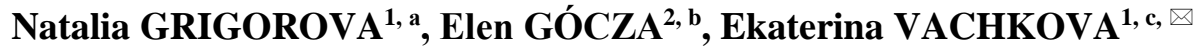 \\ ${ }^{1}$ Trakia University, Faculty of Veterinary Medicine, Department of Pharmacology, Animal Physiology and Physiological Chemistry, \\ Stara Zagora, Bulgaria; ${ }^{2}$ Applied Embryology and Stem Cell Research Group, Department of Animal Biotechnology, Gödöllö, Hungary \\ a ORCID: 0000-0001-5749-7773; ' ORCID: 0000-0001-7720-4720; 'ORCID: 0000-0002-9291-332X \\ Corresponding author: katvach@gbg.bg \\ Received date: 31.01 .2020 - Accepted date: 05.06.2020
}

\begin{abstract}
Cardiovascular diseases are still one of the most common reasons for mortality in humans. Mesenchymal stem cells (MSCs) are preferable in cardiac regeneration cell-based therapies because of their allogeneic and high proliferative potential. The electrophysiological properties of the rabbit heard is closer to human than the mouse. The current study aimed to trace mRNA expression changes of two stemness/cardiogenic differentiation ability-related transcriptionala factors OCT4 and GATA4 in rabbit MSCs during early stages of induced cardiomyocyte differentiation in vitro. The mesenchymal stem cell originated from different anatomical areas-subcutaneous, visceral, bone marrow and pericardial tissue. The cardiac differentiation protocol for mouse embryonic stem cells in hanging drop was adopted in rabbit MSCs. The best formed embryonal bodies (EBs) like structures were collected and cultivated on gelatin-coated plates. The total mRNA was obtained before cardiac differentiation and on the 6th day after it. SYBER based real-time PCR was performed to evaluate the mRNA expression fold-changes of OCT4 and GATA4. The cultivation of MSCs in hanging drops during cardiac differentiation induced EBs formation, without any contractile activity up to the 6th day of the differentiation in all cell types. The applied differentiation protocol significantly downregulated GATA4 expression in ADSCs - EBs, while in BMSCs, both target genes were significantly upregulated. In conclusion, the adopted cardiac differentiation protocol from mouse embryonic stem cells could be a useful approach for rabbit bone marrow mesenchymal stem cells. Since the rest of the cells revealed weak cardiogenic capability at this early stage, some modifications of induction protocols should be considered.
\end{abstract}

Keywords: Cardiogenic differentiation, mesenchymal stem cells, rabbit.

\section{Introduction}

In 2016 World Health Organization (WHO) launched a new initiative called "Global Hearts" to prevent and fight cardiovascular diseases (CVDs), which cause 17.9 million people to die each year (or $31 \%$ of all deaths worldwide). Despite all efforts, heart attacks, and strokes still are the world's leading causes of death (1). CVDs are usually associated with the loss of nonregenerative, terminally differentiated cardiomyocytes (5). Thus leads to functional disability of heart tissue which raises the necessity of restoring the primary myocardial cell's properties-conduction of action potential and contraction. The most used option is organ transplantation, but this approach has a variety of limitations. Cardiovascular regenerative therapy proposes some approaches as cellular therapy and stem cells application. Resolving related problems includes several factors from the improvement of cellular microenvironment up to new organ development.

During the last decade, the transgenic mouse became a very common and widely used experimental model for studying many diseases in humans including cardiovascular disorders. Still, comparing their electrophysiological properties murine hart reveals some disadvantages as shorter electrocardiographic RR intervals, higher heart rates (600 beats/min) (35) and different repolarization pattern (24) manifested as shorter action-potential (AP) waveforms, lack of plateau phases, unclear ST segments and T-waves (23). In contrast, the heart rate of the laboratory rabbit (Oryctolagus cuniculus) is slower than in mice and enables tools developed for the evaluation of human cardiac function (18).

Nowadays, stem cell therapy is successfully used for the treatment of leukemias, anemias, immunodeficiencies, healing severe burns, corneal defects, neurodegeneration and myocardial infarction $(7,8)$. Among all types of MSCs in adult animals and humans, subcutaneous depots of white adipose tissue are the most used source because it is abundant and easily accessible $(2,20)$. MSCs and adipose-derived stem cells (ADSCs) in particular, are preferable as well because of their allogeneic high proliferative potential. They have been applied in cases of 
osteoarthritis, diabetes mellitus, heart disease, soft tissue regeneration, reconstructive surgery after mastectomy and facial repair $(20,21,29)$. Recently the scientists focused on another subtype of ADSCs, which anatomically lay closer to the myocardium and because of their ability of migration could be a promising source of self-renewing and reparation in cases of heart attacks and strokes. There are variations in MSCs differentiation capability concerning its origin. For example, in human epicardial ADSCs revealed higher cardiomyogenic potential when compared with the pericardial and omental ADSC subtypes (32).

Taking aforementioned together, in the current study we aimed to elucidate whether the isolated primary rabbit MSCs (adipose-derived stem cells from subcutaneous, visceral and pericardial tissue, and bone marrow-derived stem cells) possess any capability to differentiate into cardiomyocytes by monitoring mRNA expression of two stemness/cardiac-specific genes-OCT4 and GATA4 respectively. Because of their functional similarity to humans, those cells could be a more appropriate object for experimental models design, mimicking cardiac malfunctions and benefit to the development of more effective drugs.

\section{Material and Methods}

Isolation and expansion of rabbit MSCs: Three 28days-old New Zealand white rabbits were used for MSCs isolation. All procedures, concerning tissue samples obtaining, were approved and performed according to the recommendations of the Ethics Committee at the Faculty of Veterinary Medicine of Trakia University, Stara Zagora (N56/17.05.2013). The animals were euthanized, the femoral aspiration of bone marrow was performed, and samples with an approximate weight of 1-1.5g each, were collected from the subcutaneous (interscapular region), and pericardial adipose tissue. Fibroblasts from kidney were also isolated to serve as a negative control. Isolation of stromal vascular fraction cells (SVF) was performed as described by Vachkova et al. (30). Briefly, the samples were cut into small pieces and collagenase digestion was applied for 2 hours. After double washing and centrifugation at $300 \mathrm{G}$ for $10 \mathrm{~min}$., the pellet was resuspended and seeded in tissue flasks. Further, the isolated cells were incubated in basal medium consisted of DMEM (Cat. D5546), 10\% FBS (Cat. F7524), $20 \mathrm{ml} / \mathrm{L} \mathrm{L}-$ glutamine (Cat. G7513) and $10 \mathrm{ml} / \mathrm{L}$ antibiotic mixed solution (pen/strep/amphoB, Cat. A5955) all from Sigma (St. Louis, MO, USA) and kept in a humidified $5 \% \mathrm{CO}_{2}$ condition at $38^{\circ} \mathrm{C}$ until reaching $90-95 \%$ confluence. In addition, twenty-four hours after the initial plating, supernatant suspension from subcutaneous adiposederived stem cells (ScADSCsS1) was collected, and seeded under the above-described conditions.

Finally, the following experimental MSCs groups were formed: subcutaneous adipose-derived stem cells
(ScADSCs) and its supernatant (ScADSCsS1); bone marrow mesenchymal stem cells (BMSCs); pericardial adipose-derived stem cells (PcADSCs) and the negative control group - fibroblasts from rabbit kidney (NC-FKR).

Multilineage differentiation potential of described above MSCs was verified by tri-lineage differentiation assays, as described by Vachkova et al. (30).

Cardiac differentiation in hanging drop: For cardiac differentiation, the hanging drop method $\left(20 \mu \mathrm{l} / \mathrm{drops}, 2 \times 10^{4}\right.$ cells $\left./ \mathrm{ml}\right)$ was used as described by Wobus et al. (31). To form embryoid bodies (EBs), rabbit mesenchymal cells were cultured in hanging drops with Iscove's Modified Dulbecco's Medium (Cat. 12440053, IMDM, Invitrogen) supplemented with $20 \% \mathrm{FCS}, 50 \mu \mathrm{g} / \mathrm{ml}$ streptomycin (Cat. S9137, SIGMA), 50U/ml penicillin (Cat. P3032, SIGMA), 0,1 mM 2-mercaptoethanol (Cat. M7522, SIGMA), glutaMax (Cat. 35050061, Gibco, 100x), nonessential amino acids (Cat. 11140050, NEAA, Gibco, 100x) and $100 \mu \mathrm{M}$ monothioglycerol (Cat. M6145,

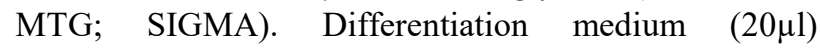
containing 400 MSCs was placed on the lids of Petri dishes filled with phosphate-buffered saline (Cat. 14190144, Invitrogen, PBS). In several hours, cell aggregates appeared, and after two days in hanging drops, embryoid bodies observed. EBs were cultured than in suspension for three days and finally, they were separately transferred onto gelatin-coated 24-well culture plates for five days. During the cultivation, microscopic observation of spontaneously contractile activity was performed daily.

Gene expression assays: The real-time PCR analysis was conducted before and at day 5 of the cardiac differentiation (an early differentiation phase). The total mRNA was isolated using the TRI Reagent kit (MRC, UK) according to the manufacturer's instructions. The concentration of the extracted RNA was determined using the NanoDrop Spectrophotometer. The isolated RNA was stored in $-70^{\circ} \mathrm{C}$ and the first-strand cDNA was synthesized by High capacity cDNA-reverse-transcription Kit (Cat. 4368814, Applied-Biosystems, Life Technologies, Carlsbad, US) following the instructions of the manufacturer and the cDNA was stored at $-20^{\circ} \mathrm{C}$. Thereafter the cDNA was used for quantitative real-time PCR, using the SYBR Green PCR master mix (Cat. 4309155, Applied Biosystems, Life Technologies, Carlsbad, US). The primers for target genes were as follows: OCT4 (Octamer-binding transcription factor 4, F:TGGGTGGAGGAAGCTGACAAC, R:ACTGGTTC GCTTTCTCTTTCGG) and GATA4 (GATA binding protein 4; F: CCGGAGCCTACGAGACGGGT, R: GGCGTGGGCACATAGACCGG) (19).

Statistical analysis: All statistical analyses were performed using Statistica v.10 (StatSoft Inc., 19842011). The raw data was normalized to the housekeeping gene - GAPDH (Glyceraldehyde 3-phosphate dehydrogenase; F: GCCGCTTCTTCTCGTGCAG, R: 
ATGGATCATTGATGGCGACAACAT) (22) and then the relative expression was calculated by the $\Delta \Delta \mathrm{Ct}$ method. Rabbit fibroblasts isolated from kidney (NCFKR) were used as a control group. Further MannWhitney $U$ test was performed to assess the statistical differences between the non-differentiated and early-stage differentiated MSCs (EBs). Results are presented as the mean \pm standard error of the mean (mean \pm SEM). The statistical significance of the differences was accepted at $\mathrm{P}<0.05$.

\section{Results}

Embryoid bodies formation in hanging drops: The cardiac differentiation of MSCs in hanging drops causes the forming of three-dimensional aggregates called embryoid bodies within two days. Most of the newly formed EBs were asymmetric (Figure $1-A, B$ and C). They do not exhibit contractile activity up to the $6^{\text {th }}$ day of the differentiation. The best-formed EBs were established in PcADSCs (Figure1-D). The negative control group kidney fibroblasts (NC-FKR) did not form EBs (Figure 1-E).

Relative changes in OCT4 mRNA expression levels: All rabbit cell types from the current experiment expressed the pluripotency gene - Octamer-binding transcription factor 4 , but the relative mRNA expression was close in ScADSCsS1 and BMSCs or even downregulated in ScADSCs and PcADSCs; $\mathrm{P}<0.01$, compared to the NC-FKR. After initial cardiogenic differentiation and EBs formation, the OCT4 mRNA expression was upregulated in BMSCs_EBs $(\mathrm{P}<0.01)$ compared to NC-FKR and BMSCs $(\mathrm{P}<0.01)$. The mRNA expression of OCT4 was downregulated related to the negative control $(\mathrm{P}<0.01)$ in the rest MSCs_EBs types (Figure 2-A).

Relative changes in GATA4 mRNA expression levels: GATA4 mRNA expression (Figure. 2-B) before the cardiac differentiation was downregulated in ScADSCs, ScADSCsS1, and BMSCs $(\mathrm{P}<0.01)$ and upregulated in PcADSCs than in NC-FKR $(p<0.01)$. After initial cardiogenic differentiation and EBs formation, a slight increase in GATA4 mRNA expression was observed in ScADSCs_EB and ScADSCsS1_EB compared to their relative undifferentiated groups $(\mathrm{P}<0.05)$. However, they still remained downregulated when compared to the NC-FKR $(\mathrm{P}<0.01)$. The observed gene expression was considerably enhanced in BMSCs_EBs related to ScADSCs_EBs and ScADSCsS1_EB $(\mathrm{P}<0.05)$. A similar pattern was established when the results from BMSCs_EBs were compared to its undifferentiated control (BMSCs), PcADSCs_EBs and NC-FKR, but the differences were statistically insignificant. Surprisingly, GATA4 mRNA expression in PcADSCs_EBs was fourfold suppressed compared to PcADSCs $(\mathrm{P}<0.05)$ and the values reached those of the negative control (NCFKR). Additional tri-lineage differentiation assays (osteogenic, chondrogenic and adipogenic) was successfully performed in order to verify the multipotent properties of the isolated cells as described Vachkova et al. (30).

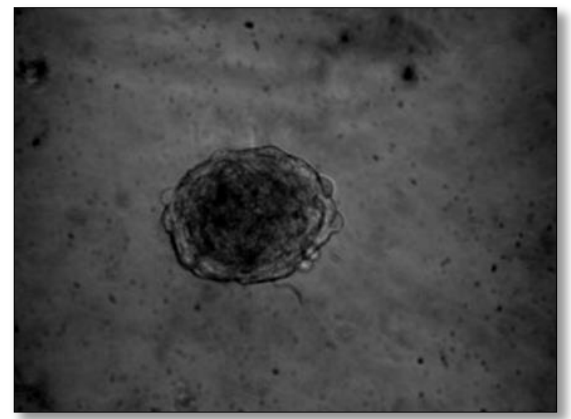

A) ScADSCs_EB

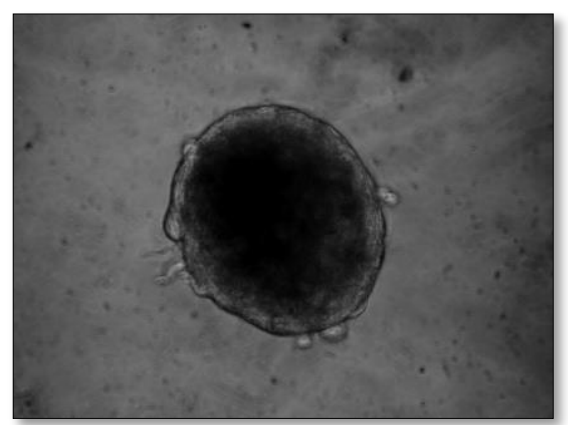

D) PCADSCs_EB

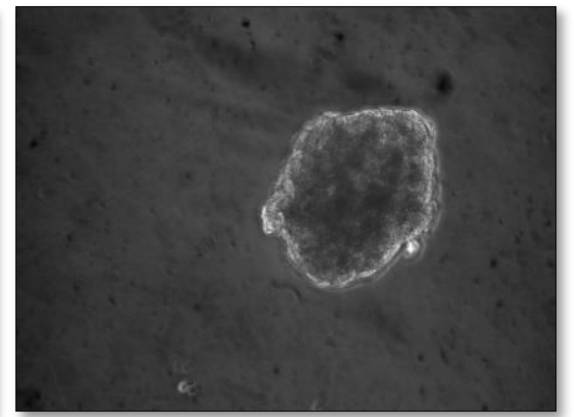

B) SCADSCSS1_EB

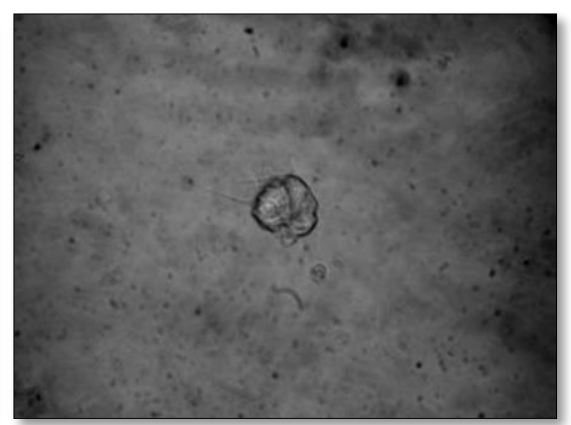

E) NC-FKR (negative control)

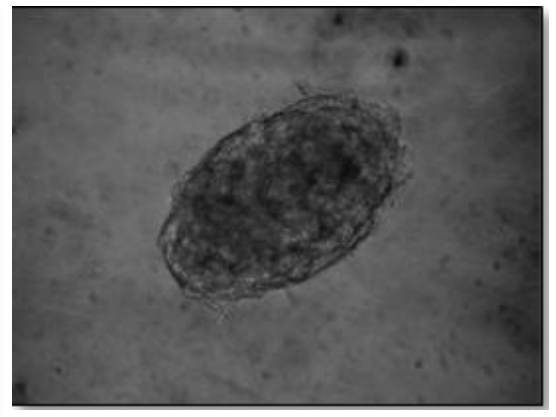

C) BMSCS_EB

Figure 1. Embryoid bodies (EB) formation from different cell types during cardiogenic differentiation in hanging drops $(\mathrm{x} 4)$.

Most of the EBs were asymmetric (A, B and C). The best-formed EBs were established in PcADSCs (D) since the negative control group - kidney fibroblasts (NC-FKR) did not form EBs (E). 


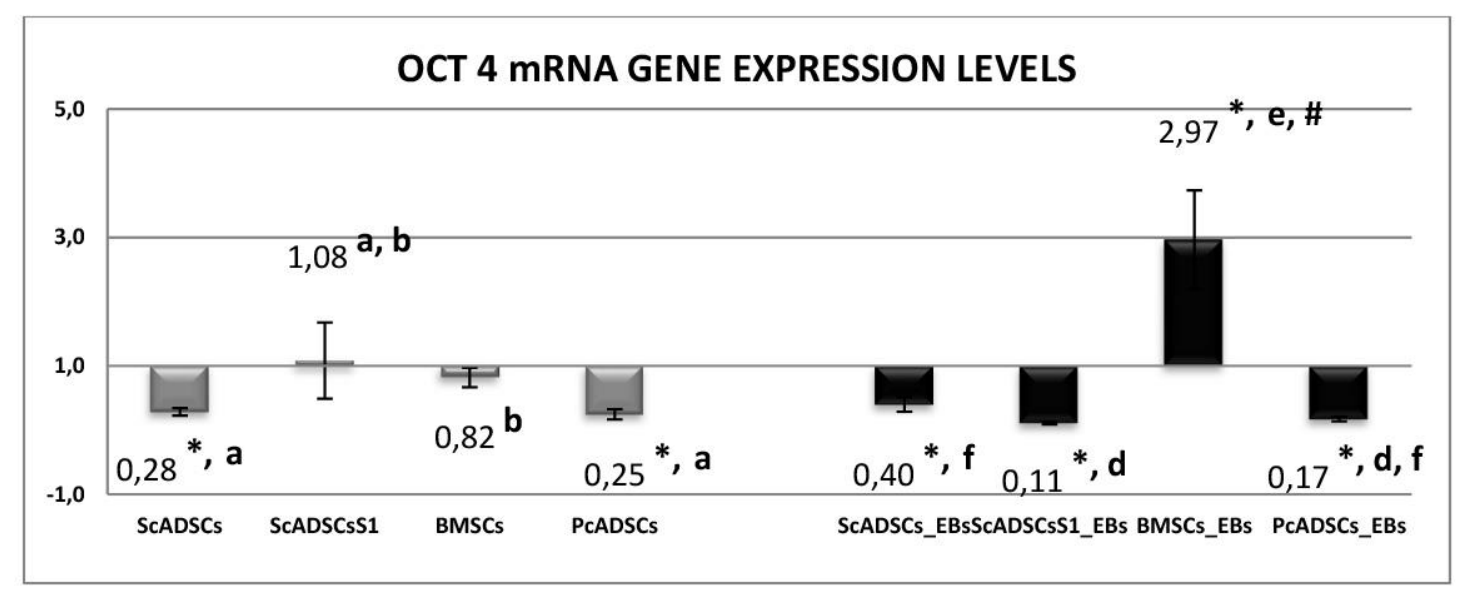

A

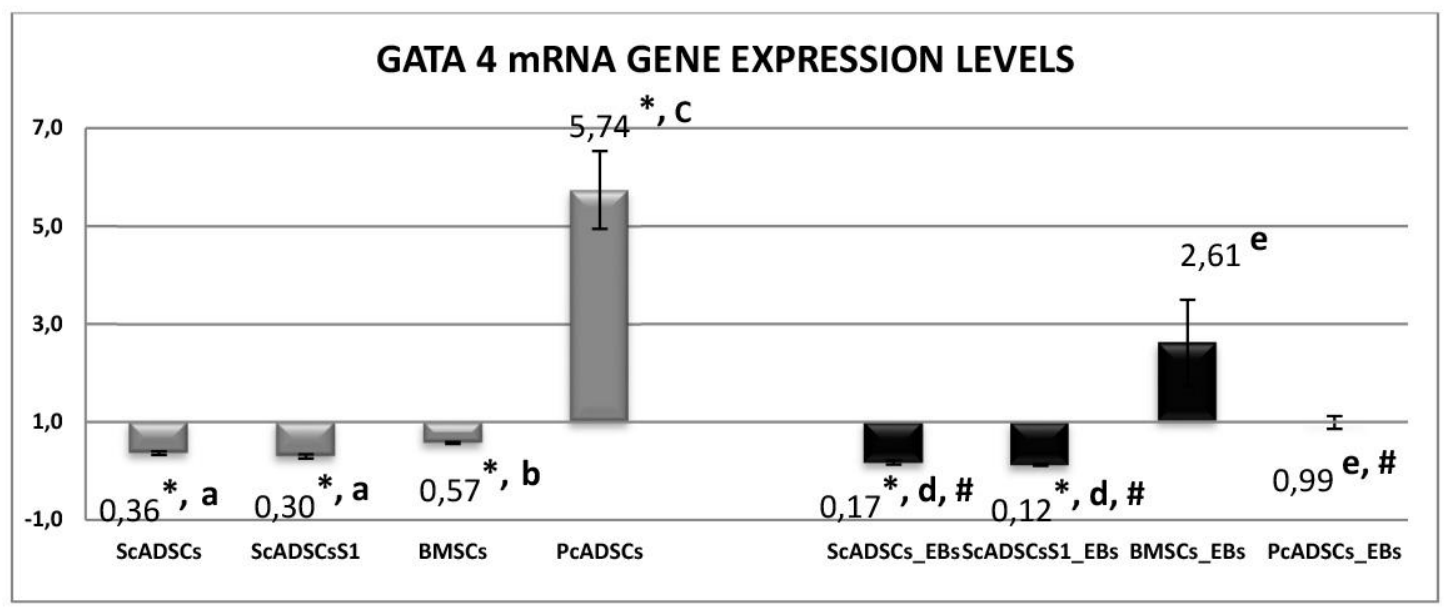

B

Figure 2. Relative changes in OCT4 (A) and GATA4 (B) mRNA expression levels before differentiation and after cardiac differentiation in MSCs.

The results are presented relatively to the negative control group (rabbit fibroblasts from the kidney) and the asterisk indicates a significant difference between the negative control and each MSCs or MSCs_EBs type $(\mathrm{P}<0.01)$. The different letters indicate statistically significant differences among the MSCs types, as follows: the letters "a", "b" and "c"- before cardiac differentiation $(\mathrm{P}<0.01)$ and the letters "d", "e" and " $\mathrm{f}$ " - after cardiac differentiation $(\mathrm{P}<0.05)$. The symbol \# indicates the significant differences within the same MSCs type, before and after cardiac differentiation $(\mathrm{P}<0.01)$.

\section{Discussion and Conclusion}

The presented study followed the mRNA expression of two transcriptional factors related to the stemness/cardiac differentiation- OCT4 and GATA4, respectively, by using quantitative RT-PCR to evaluate the differentiation potential of rabbit MSCs. From all tested mesenchymal stem cell types, our results showed that the MSCs, isolated from bone marrow, possess the best cardiogenic differentiation potential because both observed transcriptional factors were upregulated after cardiogenic induction. In general, the function of OCT 4 is related to the regulation of MSCs' multipotent properties (12). The GATA4 is a transcriptional factor, which together with others, such as Nkx2-5 and Tbx5, promotes cardiac morphogenesis during the embryonal stage and maintains cardiac function in the adult heart, regulating its hypertrophic growth and cardiomyocytes survival (26). Recently have been reported a mechanism that resembles the reprogramming process of adult stem cells in which OCT4 expression is directly related to the ability of MSCs to acquire a partial cardiomyocyte phenotype. Accordingly, MSCs must first gain OCT4 (dedifferentiate) before being able to commence cardiomyocyte reprogramming (33). Considering the early stage of cardiogenic differentiation in our study, the obtained results for bone marrow-derived MSCs after cardiogenic induction corresponded to the mechanism mentioned above. This might be the reason why cardiogenic induction provokes the upregulation of both target genes in this group.

In humans, subcutaneous fat tissue seemed to be better source of MSCs than bone marrow (36). In contrast, 
in our study the mRNA expression of OCT4 and GATA4 from subcutaneous and visceral adipose tissue MSCs, were not affected by cardiogenic induction conditions, or even were particularly downregulated. There are reports emphasizing that OCT4 was not expressed in cultured human adult MSCs achieved from bone marrow, adipose tissue, and heart, and only NANOG expression was detected after in vitro culture (27). Obviously, the applied protocols in our study were more suitable for cardiogenic induction in bone-marrow derived MSCs in rabbits. A possible reason could be disability of the MSCs achieved from adipose tissue to switch OCT4 de-differentiation mechanism and proceed to the cardiomyocyte reprogramming. Culture medium composition could also influence the expression of stem cell-related transcriptional factors, including Nanog, OCT4, Sox-2, and Rex-1 $(3,4)$. Following this direction, another possible test for future improvement of cardiogenic induction protocols in rabbits cold be pre-treatment with a DNA demethylating agent 5-azacytidine (5-aza) and co-culture with neonatal rat cardiomyocytes (6) or using fibroblast cell as feeder layer which could enhance the expression of key differentiation genes (9) and to maintain the pluripotent state of stem cells (25). Recently was reported a new approach in benefiting proper cardiomyocyte function by using of highly specialized myocardial matrix hydrogel, which enhances human cardiac progenitor cells cardiogenic potential, proliferation, and survival (11).

The most surprising result of the current study was observed in pericardial MSCs, where the highly expressed GATA 4 was downregulated by cardiogenic induction. Anatomically the pericardial fat tissue is lining above the fibrous membrane of the pericardium and differs from the epicardium by its embryonal origin, blood supply and biochemical properties $(13,28)$. Up to date, a little is known about the multipotent properties of pericardial MSCs. Most of the reported studies are focused on the hydrogel embedded implantation of a new form of adult cardiac stem cells, as called cardiospheres (CSPs), into the pericardial cavity $(16,34)$ or using the de-cellularized pericardium membrane as a scaffold for MSCs culturing $(10,14)$. The CSPs is a new approach of stem cell therapy for myocardial infarction treatment, using the ability of those cells to differentiate into cardiomyocytes in vivo (34). Since those stem cells are not able to penetrate and reach up to the myocardium because of the double-walled structure of the pericardium, only proteins with a molecular mass below $40 \mathrm{kDa}$ can diffuse (17) and this allows paracrine communications within them. In that sense, based on the high expression of GATA4 before and its downregulation by cardiogenic induction, together with constantly low OCT4 expression, we could suspect that the isolated MSCs from pericardium fat tissue might be particularly differentiated. Therefore, the functional purpose of those cells is to serve as a source of paracrine mediators and influence the myocardial tissue regeneration by producing growth factors in the pericardial cavity (34).

Since ASCs may differ between the origin sites, biological characteristics, and secreted trophic factors (15), the observed differences in our study are not surprising. The adopted from mouse embryonic stem cells protocol for in vitro cardiac differentiation in hanging drops is more suitable to the bone marrow MSCs in rabbits than for the rest of the groups. Microscopically, all isolated cell types, excluding negative controls, were capable of forming asymmetric EBs even none of them were able to contract spontaneously. It is a good early sign for the cardiogenic differentiation potential of those cells and with some modifications in induction protocol, according to sites of ADSCs origin, better results could be expected.

Nevertheless, the current study is preliminary research on cardiogenic differentiation ability of rabbit MSCs, isolated from subcutaneous, visceral and pericardial adipose tissue, and bone marrow. In conclusion, basing on the reported data, our study reveals that in those conditions (chemical composition, compound concentrations into the induction media, duration, feeding layer, the anatomical origin of the cells, etc.), the most of the isolated cell types revealed a weak capability to differentiate into cardiomyocytes. Even so, this protocol could be a useful approach for bone-marrow-derived MSCs after cardiac differentiation in rabbits where the most definite cardiogenic potential was established at this early stage of differentiation.

\section{Financial Support}

Funded by Project 4/13 within Trakia UniversityFVM; Scholarship (Dr. Natalia Grigorova) within STSM COST Action TD1101-RGB- Net, Dr. Zsuzsanna Bősze and Dr. Elen Gócza, Applied Embryology and Stem Cell Research Group of Institute for Animal Biotechnology, Gödöllö, Hungary.

\section{Ethical Statement}

This study was approved by Ethics Committee at the Faculty of Veterinary Medicine of Trakia University, Stara Zagora (N56/17.05.2013).

\section{Conflict of Interest}

The authors declared that there are no conflict of interest.

\section{References}

1. Anonymous (2017):World Health Organization (WHO). Available at: https://www.who.int/en/news-room/factsheets/detail/cardiovascular-diseases-(cvds). (Accessed 17 May 2017). 
2. Baer PC (2014): Adipose-derived mesenchymal stromal/stem cells: An update on their phenotype in vivo and in vitro. World J Stem Cells, 6, 256-65.

3. Baer PC, Geiger H (2012): Adipose-derived mesenchymal stromal/stem cells: tissue localization, characterization, and heterogeneity. Stem Cells Int, 2012, 812693.

4. Baer PC, Griesche N, Luttmann W, et al (2010): Human adipose-derived mesenchymal stem cells in vitro: evaluation of an optimal expansion medium preserving stemness. Cytotherapy, 12, 96-106.

5. Burridge P, Keller G, Gold JD, et al (2012): Production of de novo cardiomyocytes: human pluripotent stem cell differentiation and direct reprogramming. Cell Stem Cell, 10, 16-28.

6. Choi YS, Dusting GJ, Stubbs S, et al (2010): Differentiation of human adipose-derived stem cells into beating cardiomyocytes. J Cell Mol Med, 14, 878-889.

7. Conrad C, Huss R (2005): Adult stem cell lines in regenerative medicine and reconstructive surgery. J Surg Res, 124, 201-208.

8. Dulak J, Szade K, Szade A, et al (2015): Adult stem cells: hopes and hypes of regenerative medicine. Acta Biochim Pol, 62, 329-37

9. Ejaz A, Hatzmann FM, Hammerle S, et al (2019): Fibroblast feeder layer supports adipogenic differentiation of human adipose stromal/progenitor cells. Adipocyte, $\mathbf{8}$, 178-189.

10. Ferroni L, Gardin C, Bellin G, et al (2019): Bovine pericardium membrane as new tool for mesenchymal stem cells commitment. J Tissue Eng Regen Med, 13, 1805-1814.

11. Gaetani R, Yin C, Srikumar N, et al (2016): Cardiacderived extracellular matrix enhances cardiogenic properties of human cardiac progenitor cells. Cell Transplantation, 25, 1653-1663.

12. Greco SJ, Liu K, Rameshwar P (2007): Functional similarities among genes regulated by OCT4 in human mesenchymal and embryonic stem cells. Stem Cells, 25, 3143-3154.

13. Iacobellis G, Corradi D, Sharma AM (2005): Epicardial adipose tissue: anatomic, biomolecular and clinical relationships with the heart. Nature Clinical Practice Cardiovascular Medicine, 2, 5365-43.

14. Khorramirouz R, Go JL, Noble C, et al (2019): In vivo response of acellular porcine pericardial for tissue engineered transcatheter aortic valves. Sci Rep, 9, 1094.

15. Kocan B, Maziarz A, Tabarkiewicz J, et al (2017): Trophic activity and phenotype of adipose tissue-derived mesenchymal stem cells as a background of their regenerative potential. Stem Cells Int, 2017, 1653254.

16. Ladage D, Turnbull IC, Ishikawa K, et al (2011): Delivery of gelfoam-enabled cells and vectors into the pericardial space using a percutaneous approach in a porcine model. Gene Therapy, 18, 979-985.

17. Limana F, Bertolami C, Mangoni A, et al (2010): Myocardial infarction induces embryonic reprogramming of epicardial c-kit(+) cells: role of the pericardial fluid. $\mathbf{J}$ Mol Cell Cardiol, 48, 609-618.

18. Major P, Baczkó I, Hiripi L, et al (2016): A novel transgenic rabbit model with reduced repolarization reserve: long $Q T$ syndrome caused by a dominant-negative mutation of the KCNE1 gene. B J Pharmacol, 173, 2046-61.
19. Maraghechi P, Hiripi L, Tóth G, et al (2013): Discovery of pluripotency-associated microRNAs in rabbit preimplantation embryos and embryonic stem-like cells. Reproduction, 145, 421-37.

20. Minteer D, Marra KG, Rubin JP (2013): Adipose-derived mesenchymal stem cells: biology and potential applications. Adv Biochem Eng Biotechnol, 129, 59-71.

21. Naderi N, Combellack EJ, Griffin M, et al (2017): The regenerative role of adipose-derived stem cells (ADSC) in plastic and reconstructive surgery. Int Wound J, 14, 112124.

22. Navarrete SA, Ramin N, Tonack S, et al (2008): Cell lineagespecific signaling of insulin and insulin-like growth factor I in rabbit blastocysts. Endocrinology, 149, 515-524.

23. Nerbonne JM (2004): Studying cardiac arrhythmias in the mouse - a reasonable model for probing mechanisms? Trends Cardiovasc Med, 14, 83-93.

24. Nerbonne JM, Kass RS (2005): Molecular physiology of cardiac repolarization. Physiol Rev, 85, 1205-1253.

25. Pekkanen-Mattila M, Ojala M, Kerkelä E, et al (2012): The effect of human and mouse fibroblast feeder cells on cardiac differentiation of human pluripotent stem cells. Stem Cells Int, 2012, 875059.

26. Perrino C, Rockman HA (2006): GATA4 and the two sides of gene expression reprogramming. Circ Res, 98, 715-6.

27. Pierantozzi E, Gava B, Manini I, et al (2011): Pluripotency regulators in human mesenchymal stem cells: expression of NANOG but not of OCT-4 and SOX-2. Stem Cells, 20, 915-23.

28. Sacks HS, Fain JN (2007): Human epicardial adipose tissue: a review. Am Heart J, 153, 907-917.

29. Sanina C, Hare JM (2015): Mesenchymal stem cells as a biological drug for heart disease: where are we with cardiac cell-based therapy? Circ Res, 117, 229-233.

30. Vachkova E, Bosnakovski D, Yonkova P, et al (2016): Adipogenic potential of stem cells derived from rabbit subcutaneous and visceral adipose tissue in vitro. In Vitro Cell Dev Biol Anim, 52, 829-837.

31. Wobus AM, Holzhausen H, Jäkel P, et al (1984): Characterization of a pluripotent stem cell line derived from a mouse embryo. Exp Cell Res, 152, 212-219.

32. Wystrychowski W, Patlolla B, Zhuge Y, et al (2016): Multipotency and cardiomyogenic potential of human adipose-derived stem cells from epicardium, pericardium, and omentum. Stem Cell Res Ther, 7, 84.

33. Yannarelli G, Pacienza N, Montanari S, et al (2017): OCT4 expression mediates partial cardiomyocyte reprogramming of mesenchymal stromal cells. PLoS One, 12, 1-20.

34. Zhang J, Wu Z, Fan Z, et al (2018): Pericardial application as a new route for implanting stem-cell cardiospheres to treat myocardial infarction. J Physiol, 596, 2037-2054.

35. Zhang Y, Wu J, King JH, et al (2014): Measurement and interpretation of electrocardiographic $Q T$ intervals in murine hearts. Am J Physiol Heart Circ Physiol, 306, 15531557.

36. Zhu Y, Liu T, Song K, et al (2008): Adipose-derived stem cell: a better stem cell than BMSC. Cell Res, 18, 165. 